\title{
Quality of life outcomes in tracheobronchomalacia surgery
}

\author{
Joseph McGinn ${ }^{1}$, Benoit Herbert ${ }^{2}$, Andrew Maloney ${ }^{2}$, Byron Patton ${ }^{2}$, Richard Lazzaro \\ ${ }^{1}$ Department of Surgery, General Surgery Residency, North Shore-LIJ, Northwell Health System, Manhasset, NY, USA; ${ }^{2}$ Department of Thoracic \\ Surgery, Lenox Hill Hospital, Northwell Health System, New York, NY, USA \\ Contributions: (I) Conception and design: R Lazzaro, B Patton; (II) Administrative support: R Lazzaro, B Patton; (III) Provision of study materials or \\ patients: R Lazzaro, B Patton; (IV) Collection and assembly of data: J McGinn, B Herbert, A Maloney; (V) Data analysis and interpretation: None; (VI) \\ Manuscript writing: All authors; (VII) Final approval of manuscript: All authors. \\ Correspondence to: Richard Lazzaro. Department of Thoracic Surgery, Lenox Hill Hospital, Northwell Health System, New York, NY, USA. Email: \\ lazzaro.richard@gmail.com.
}

\begin{abstract}
Tracheobronchomalacia (TBM) is an obstructive airway disease characterized by laxity and redundancy of the posterior membrane of the main airways leading to dynamic airway collapse during exhalation. The gold standard for diagnosis is dynamic computed tomography (DCT) scan and dynamic flexible bronchoscopy (DFB). Patients with complete or near-complete collapse ( $>90 \%$ reduction in crosssectional area) of the airway are possible candidates for surgical management. Central airway stabilization by tracheobronchoplasty (TBP) effectively corrects malacic airways and has demonstrated significant improvement in objective functional measures, which is often but not uniformly accompanied by equal improvement in health-related quality of life (HRQOL) metrics. This article reviews HRQOL instruments used to report outcomes after TBM surgery.
\end{abstract}

Keywords: Tracheobronchomalacia (TBM); tracheobronchoplasty (TBP); health-related quality of life (HRQOL); tracheomalacia; bronchomalacia

Submitted Jan 02, 2020. Accepted for publication Feb 20, 2020.

doi: $10.21037 /$ jtd.2020.03.08

View this article at: http://dx.doi.org/10.21037/jtd.2020.03.08

\section{Introduction}

Excessive central airway collapse (ECAC) is characterized by a dynamic flow obstruction of the airways during exhalation. Tracheobronchomalacia (TBM) and excessive dynamic airway collapse (EDAC) are two distinct airway pathologies that encompass ECAC. EDAC is characterized by atrophy of the muscular fibers in the posterior membrane of the airway. During exhalation this lax, redundant posterior membrane bulges anteriorly and narrows the airway. TBM is characterized by softening and weakness of the anterior tracheobronchial cartilaginous structures that can also lead to posterior membrane invagination (1). Since both entities are similar in presentation, diagnostic evaluation, and therapeutic approach, TBM will be used to refer to both.

TBM can be classified into congenital and acquired forms. The congenital form is predominantly observed in the pediatric population and is beyond the scope of this review. The precise pathophysiological etiology of acquired TBM is not known, however it appears in the context of chronic airway inflammation such as in patients with chronic obstructive pulmonary disease (COPD), relapsing polychondritis, tracheostomy, or chronic inhaled corticosteroid use (2-5). On presentation it can involve the trachea alone or one or both mainstem bronchi in which case it is considered diffuse. Progression towards severe diffuse TBM is the fate of most patients. A bronchoscopic surveillance study of patients with tracheomalacia and bronchomalacia revealed a rate to severe diffuse TBM of $67 \%$ and $100 \%$ respectively over a 5 -year follow-up $(6,7)$.

\section{Symptoms and presentation}

The symptoms of TBM are nonspecific and mimic common respiratory conditions. For this reason, TBM has been underrecognized as a cause for airway disease and thus its 
true incidence is not known. TBM has previously been reported in $4.5 \%$ of the general population (8). Ikeda et al. demonstrated that over $13 \%$ of patients undergoing evaluation for respiratory complaints were found to have TBM (9). Patients exhibit dyspnea on exertion, persistent cough, barking cough (due to the vibration of a redundant collapsing posterior membrane against the anterior wall), syncope due to coughing episodes, expiratory stridor/ wheeze, inability to clear secretions, and predisposition to respiratory infections. High clinical suspicion is warranted for patients that exhibit symptoms that are out of proportion to respiratory dysfunction caused by more common diseases such as COPD and asthma, or if symptoms fail to respond to standard medical interventions for such illnesses. For some, the diagnosis is only first considered after acute presentation with respiratory failure or failure to wean from ventilatory support as the endotracheal tube and positive pressure stent the airways open. Others with long-term refractory symptoms prompt further investigation with the two established diagnostic standards: dynamic computed tomography (DCT) and dynamic flexible bronchoscopy (DFB). Both modalities provide visualization of airway collapse during exhalation that can then be quantified. The cutoff of $\geq 70 \%$ narrowing on forced expiration is the recognized threshold for diagnosing TBM on DCT and is further subclassified into mild ( $70 \%$ to $80 \%$ ), moderate ( $81 \%$ to $90 \%)$, and severe (>90\%).

\section{Indications for tracheobronchoplasty (TBP)}

Initial treatment for patients suspected of TBM entails supportive measures and maximum medical treatment of comorbidities, such as COPD, asthma, and obstructive sleep apnea. Part of the workup for TBM is ensuring that symptoms cannot be reasonably explained by the presence of other more common respiratory illnesses. Patients should be evaluated and treated for vocal cord dysfunction and gastroesophageal reflux disease (GERD). The prevalence of GERD in patients with TBM has been reported at $43 \%$ and of those, $46 \%$ report an improvement in respiratory symptoms with maximal medical treatment or anti-reflux surgery and require no further TBM treatment. If left untreated, GERD may negatively impact outcomes after TBM surgery (10). Continuous positive airway pressure (CPAP) ventilation can improve symptoms and exercise tolerance by stenting open airways but shows no long-term benefit and is generally regarded as a "bridge" to surgery or a final treatment for patients who are not surgical candidates
$(11,12)$.

TBP is the surgical management of TBM; it entails the reshaping of the trachea into the more physiologic " $\mathrm{D}$ " shape and splinting of the posterior wall of the airway using a polypropylene mesh applied from the thoracic inlet to the distal left mainstem bronchus and distal bronchus intermedius. TBP has been described using an open right thoracotomy approach or a minimally invasive robotic approach. Airway stents have been used preoperatively to predict which patients will benefit most from TBP. A Y-shaped silicone or nitinol tracheobronchial stent is deployed in portions of the airway that would be stabilized by TBP. While it is reported that a significant proportion of these patients on a "stent trial" exhibit improvement in symptoms and a successful stent trial appears to predict improvement after TBP, airway stents also induce mucus plugging, increased cough, increased secretions, and are subject to migration $(13,14)$. These complications limit the ability of the "stent trial" to reliably predict which patients will respond to TBP. For this reason, stent trials are no longer practiced by our institution.

\section{Baseline and interval assessment}

Patients with severe or complete collapse of the trachea as evidenced on DCT or DFB should be considered for TBP, however, objective measures of airway collapse and pulmonary dysfunction alone are not sufficient to indicate surgery. Previously, the cutoff for diagnosing TBM was $\geq 50 \%$ airway narrowing during exhalation. However, one study revealed that $70 \%$ to $80 \%$ of healthy asymptomatic patients exceeded $>50 \%$ expiratory reduction in crosssectional area on DCT (2). Furthermore, pulmonary function testing (PFT) via spirometry is not additive to the diagnostic workup for TBP candidacy and the degree of airway collapse does not correlate with spirometry measures such as forced expiratory volume at one second (FEV1). Up to $21 \%$ of patients with moderate to severe TBM reveal normal flow-volume loops on spirometry (15). Gangadharan et al. reported on 63 patients undergoing open TBP over a 7-year period. While subjective measures showed improvement in symptoms, there was no improvement in FEV1; in fact almost $40 \%$ of patients had a worse FEV1 postoperatively (16). Our institution's robotic TBP series also revealed that $30 \%$ of patients had slightly worse FEV1 despite high overall satisfaction score and alleviation of symptoms with the operation (17). These results are congruent with the findings of a study by Lee $e t$ al. that 
revealed that symptom amelioration was independent of a decrease in air trapping as measured by CT scan, which suggests that symptom relief is not necessarily related to early expiratory flow-volume mechanics (18).

The 6-minute walk test (6MWT) is a simple assessment of functional status where a patient is asked to walk as far as they can in 6 minutes on a straight flat surface. The distance and symptoms exhibited during the test are recorded. The change in distance after a given intervention considered to be clinically significant is approximately 230 feet (19). In 2011 Gangadharan et al. reported a statistically significant improvement in 6MWT distance from an average of 987 feet preoperatively to 1,187 feet postoperatively $(n=33)$. While improvements in FEV1 and distance on the 6MWT may follow an improvement in symptomatology, they do not reliably predict efficacy of TBP preoperatively. Poor FEV1 and lower distance on the 6MWT have however been correlated with increased risk of postoperative pulmonary complications (20). Spirometry in general airway disease has only modest correlation with exercise intolerance and functional status $(21,22)$. Furthermore, spirometric data correlates poorly with disease-specific health-related quality of life (HRQOL) indices (23).

The failure of objective measures to effectively predict improvement in symptoms after surgical intervention demands that patients considering surgery for TBM undergo a baseline and interval assessment of symptomatology and quality of life (QOL). There are several validated questionnaires for airway disease that have been used to quantify the effect of TBM on QOL. While recurrent infection and cough can be observed objectively, quantifying the impact of these sequelae on QOL is historically more challenging. Even more elusive is the quantification of dyspnea. The American Thoracic Society (ATS) defines dyspnea as the subjective experience of breathing discomfort comprised of qualitatively distinct sensations that vary in intensity. The ATS created a dyspnea scale to assess an individual's subjective rating of dyspnea severity based on the magnitude of a given activity that provokes the sensation. The scale is from 0 (breathless with exercise) to 5 (too breathless to leave the house). The Medical Research Council (MRC) dyspnea scale, created by the Canadian Thoracic Society, is equivalent in both items and scoring to that of the ATS.

The ATS and MRC dyspnea scales are simple to administer and can even assist in predicting risk of death from any respiratory cause in COPD patients, however they measure only one dimension of dyspnea and the grades are broad, possibly rendering small but significant changes after interventions imperceptible (24). The baseline dyspnea index (BDI) and transitional dyspnea index (TDI) are more discerning metrics demonstrated to be more reliable and valid than the ATS dyspnea index (25). The BDI and TDI are interview-administered questionnaires consisting of 24 items organized into 3 domains (functional impairment, magnitude of task, and magnitude of effort). Each item is graded using a Likert scale from 0 (very severe) to 4 (no impairment); the lower the score the worse the severity of dyspnea. A BDI collected before a given intervention can be calculated and then compared to a TDI calculated using the same questionnaire given post-intervention. BDI ranges from 0 (most severe) to 12 (least severe) while TDI ranges from -9 to +9 indicating deterioration or improvement in severity, respectively. A change in 1 or more for TDI is considered the minimum for significant change. The BDI and TDI have been shown to be reproducible, exhibit internal consistency, and has been validated against objective measures of respiratory dysfunction as well as other frequently used subjective instruments, such as the Saint George's Respiratory Questionnaire (SGRQ) (26).

The SGRQ is a well-established disease-specific QOL instrument developed for asthma and COPD (27). The SGRQ questionnaire consists of 14 questions with weighted responses that yield scores from 0 to 100 , with higher scores indicating poorer health. This score is reported as a total and broken down into the following 3 separate scores: the symptom score addressing the frequency of symptoms, the activity score measuring the disturbance to daily activities, and the impact score which covers a range of psychosocial function. There is extensive data from both crosssectional and longitudinal studies that demonstrate the validity of using SGQR to discriminate differences between patients and evaluate changes within the same patients after intervention, respectively. A change score of 4 units is the minimum change considered to be a clinically significant improvement after intervention $(27,28)$.

Another less commonly utilized QOL instrument is the Cough Specific Quality of Life Questionnaire (CQLQ). Developed by French et al. in 2002, the CQLQ comprises a list of 28 statements that patients agree or disagree with using a 4-point Likert scale ("strongly disagree" to "strongly agree"). The statements assess the impact that a patient's cough has on their daily living (e.g., "I have been completely prevented from engaging in important activities such as work, school, or volunteer services"). When tested on 154 chronic coughers, the CQLQ demonstrated excellent 
internal consistency, test-retest reliability, and validity in determining impact of cough on patient's QOL (29).

\section{HRQOL outcomes}

Ernst $e t$ al. reported QOL results of a retrospective study on prospectively collected data between 2002 and 2009 on COPD patients with TBM undergoing both airway stent placement and TBP at Beth Israel Deaconess Medical Center (BIDMC). Outcomes were stratified using the GOLD classification of airflow limitation severity, which is based on post-bronchodilator FEV1 as percent of predicted. The improvement in ATS dyspnea scale (stent: -0.7 , TBP: -1.7 ), TDI (stent: +2.1, TBP: +3.8 ), and SGQR (stent: -8.2 , TBP: -27.6) were statistically significant when looking at the combined outcomes for all GOLD stages in both the stenting and TBP arms. Of the 94 patients that underwent the stenting trial, 29 patients underwent TBP. When performing a specific analysis on the 29 TBP patients, the improvement was still seen across the aforementioned measures but due to the small sample size this was not statistically significant. There was no significant change in 6MWT across all GOLD stages. FEV1 increased following both stenting and TBP in all groups except patients with GOLD stage I but the only statistically significant improvement in FEV1 was seen following TBP in GOLD stage 4 patients. Of note, of the 94 patients that underwent stenting, there was no significant difference in FEV1 and 6MWT after stent placement. In terms of QOL outcomes, the BIDMC group reported a significant reduction in SGQR from 74 to $46(\mathrm{P}<0.001)$ in 35 patients that underwent pre- and post-SGQR testing after open TBP. There was no evident correlation between GOLD stage and SGQR reduction.

Our institution adopted the SGQR after noting the subjective nature of outcomes during the first series of robotic TBP. Of the 15 patients that underwent pre- and postoperative SGQR, we observed a drop in median SGQR score from 65.3 preoperatively to 46.2 following robotic TBP. Of note the most improved symptom in this study was chronic cough while the least improved was shortness of breath. We continue to collect subjective data points and will publish more data utilizing SGQR in the near future.

The CQLQ QOL instrument is under-utilized for TBP but has been applied to assess symptom relief in patients undergoing a stent trial before considering TBP. Majid et al. reported in 2016 the results of a retrospective review of 33 patients with severe symptomatic ECAC who underwent 7-day placement of uncovered self-expanding metallic airway stents at BIDMC. The overall mean change between pre-stent and post-stent CQLQ was -9 ( $\mathrm{P}=0.015)$. Thirteen of these patients subsequently underwent TBP with a significantly improved CQLQ score at 3-month follow-up (30). Improvement in cough appears to be a common significant outcome after TBP across institutions further illustrating the potential utility of the CQLQ in assessing QOL outcomes for TBM patients in the future.

There are several limitations to the current QOL outcome measures utilized in the treatment of TBM. Because patients are unable to be blinded to treatment and the QOL measures are subjective, the placebo effect can possibly be overestimating the postoperative improvements. As such, the durability of symptom relief requires longitudinal follow-up. Furthermore, the study of QOL outcomes for TBP requires larger sample sizes than have been reported thus far. The largest TBP series have missing QOL data for a significant portion of patients due to late adoption of pre- and post-TBP testing thus limiting the power of these findings. Our practice continues to collect QOL data in all patients moving forward with the hope of identifying preoperative scoring patterns that reliably predict meaningful improvement for patients suffering from this debilitating disease process. Despite these limitations, the improvements in FEV1 and SGQR after maximal medical management as reported in Understanding Potential Long-term Impacts on Function With Tiotropium (UPLIFT) trial and Towards a Revolution in COPD Health (TORCH) trial are a small fraction of the reported benefits of central airway stabilization via airway stenting and especially TBP $(31,32)$.

\section{Conclusions}

As TBM becomes increasingly recognized in patient populations, so does the need for a validated prediction model for safe, efficacious intervention. The improvements in both objective functional testing and HRQOL measures after maximal medical management pales in comparison to that of central airway stabilization via airway stenting and surgery. While new techniques, such as utilization of robotic surgery, may provide more efficacious, less morbid TBM surgery, the identification of patients most likely to benefit from such techniques continues to pose a challenge. The objective measure of dynamic airway collapse with DCT and DFB are mainstays for diagnosis but are not completely predictive of improvement in symptomatology post- 
intervention. HRQOL instruments are not only important for quality assurance in the treatment of a disease whose sequelae are largely subjective, but also for research into creating predictive models for improved patient selection and prognostication.

\section{Acknowledgments}

Funding: None.

\section{Footnote}

Provenance and Peer Review: This article was commissioned by the Guest Editor (Peter J. Kneuertz) for the series "Patient reported Outcomes in Thoracic Surgery: A new Frontier" published in Fournal of Thoracic Disease. The article has undergone external peer review.

Conflicts of Interest: All authors have completed the ICMJE uniform disclosure form (available at http://dx.doi. org/10.21037/jtd.2020.03.08). The series "Patient reported Outcomes in Thoracic Surgery: A new Frontier" was commissioned by the editorial office without any funding or sponsorship. The other authors have no other conflicts of interest to declare.

Ethical Statement: The authors are accountable for all aspects of the work in ensuring that questions related to the accuracy or integrity of any part of the work are appropriately investigated and resolved.

Open Access Statement: This is an Open Access article distributed in accordance with the Creative Commons Attribution-NonCommercial-NoDerivs 4.0 International License (CC BY-NC-ND 4.0), which permits the noncommercial replication and distribution of the article with the strict proviso that no changes or edits are made and the original work is properly cited (including links to both the formal publication through the relevant DOI and the license). See: https://creativecommons.org/licenses/by-nc-nd/4.0/.

\section{References}

1. Buitrago DH, Wilson JL, Parikh M, et al. Current concepts in severe adult tracheobronchomalacia: evaluation and treatment. J Thorac Dis 2017;9:E57-66.

2. Kheir F, Majid A. Tracheobronchomalacia and excessive dynamic airway collapse: medical and surgical treatment.
Semin Respir Crit Care Med 2018;39:667-73.

3. Leong P, Tran A, Rangaswamy J, et al. Expiratory central airway collapse in stable COPD and during exacerbations. Respir Res 2017;18:163.

4. Bhatt SP, Terry NL, Nath H, et al. Association between expiratory central airway collapse and respiratory outcomes among smokers. JAMA 2016;315:498-505.

5. Husta BC, Raoof S, Erzurum S, et al.

Tracheobronchopathy from inhaled corticosteroids. Chest 2017;152:1296-305.

6. Nuutinen J. Acquired tracheobronchomalacia. A clinical study with bronchological correlations. Ann Clin Res 1977;9:350-5.

7. Nuutinen J. Acquired tracheobronchomalacia. A bronchological follow-up study. Ann Clin Res 1977;9:359-64.

8. Jokinen K, Palva T, Sutinen S, et al. Acquired tracheobronchomalacia. Ann Clin Res 1977;9:52-7.

9. Ikeda S, Hanawa T, Konishi T, et al. Diagnosis, incidence, clinicopathology and surgical treatment of acquired tracheobronchomalacia] Nihon Kyobu Shikkan Gakkai Zasshi 1992;30:1028-35.

10. Majid A, Kheir F, Alape D, et al. The prevalence of gastroesophageal reflux in patients with excessive central airway collapse. Chest 2019;155:540-5.

11. Tamura Y, Sakatani K, Yamakoshi N, et al. A case of severe COPD associated with tracheo-bronchial stenosis, treated with non-invasive positive pressure ventilation. Nihon Kokyuki Gakkai Zasshi 2008;46:915-20.

12. Adliff M, Ngato D, Keshavjee S, et al. Treatment of diffuse tracheomalacia secondary to relapsing polychondritis with continuous positive airway pressure. Chest 1997;112:1701-4.

13. Murgu SD, Colt HG. Complications of silicone stent insertion in patients with expiratory central airway collapse. Ann Thorac Surg 2007;84:1870-7.

14. Ernst A, Majid A, Feller-Kopman D, et al. Airway stabilization with silicone stents for treating adult tracheobronchomalacia: a prospective observational study. Chest 2007;132:609-16.

15. Majid A, Sosa AF, Ernst A, et al. Pulmonary function and flow-volume loop patterns in patients with tracheobronchomalacia. Respir Care 2013;58:1521-6.

16. Gangadharan SP, Bakhos CT, Majid A, et al. Technical aspects and outcomes of tracheobronchoplasty for severe tracheobronchomalacia. Ann Thorac Surg 2011;91:157480; discussion 1580-1.

17. Lazzaro R, Patton B, Lee P, et al. First series of minimally 
invasive, robot-assisted tracheobronchoplasty with mesh for severe tracheobronchomalacia. J Thorac Cardiovasc Surg 2019;157:791-800.

18. Lee KS, Ashiku SK, Ernst A, et al. Comparison of expiratory CT airway abnormalities before and after tracheoplasty surgery for tracheobronchomalacia. J Thorac Imaging 2008;23:121-6.

19. Majid A, Guerrero J, Gangadharan S, et al. Tracheobronchoplasty for severe tracheobronchomalacia: a prospective outcome analysis. Chest 2008;134:801-7.

20. Buitrago DH, Majid A, Alape DE, et al. Singlecenter experience of tracheobronchoplasty for tracheobronchomalacia: perioperative outcomes. Ann Thorac Surg 2018;106:909-15.

21. Celli BR. The importance of spirometry in COPD and asthma: effect on approach to management. Chest 2000;117:15S-9S.

22. McGavin CR, Artvinli M, Naoe H, et al. Dyspnoea, disability, and distance walked: comparison of estimates of exercise performance in respiratory disease. Br Med J 1978;2:241-3.

23. Wijnhoven HA, Kriegsman DM, Hesselink AE, et al. Determinants of different dimensions of disease severity in asthma and COPD: pulmonary function and healthrelated quality of life. Chest 2001;119:1034-42.

24. Celli BR, Cote CG, Marin JM, et al. The body-mass index, airflow obstruction, dyspnea, and exercise capacity

Cite this article as: McGinn J, Herbert B, Maloney A, Patton B, Lazzaro R. Quality of life outcomes in tracheobronchomalacia surgery. J Thorac Dis 2020;12(11):6925-6930. doi: 10.21037/ jtd.2020.03.08 index in chronic obstructive pulmonary disease. $\mathrm{N}$ Engl J Med 2004;350:1005-12.

25. Eakin EG, Sassi-Dambron DE, Ries AL, et al. Reliability and validity of dyspnea measures in patients with obstructive lung disease. Int J Behav Med 1995;2:118-34.

26. American Thoracic Society. Baseline Dyspnea Index (BDI) \& Transition Dyspnea Index (TDI). Available online: https://www.thoracic.org/members/assemblies/assemblies/ srn/questionaires/bdi-tdi.php

27. Jones PW, Quirk FH, Baveystock CM. The St George's respiratory questionnaire. Respir Med 1991;85 Suppl B:25-31; discussion 33-7.

28. Jones PW. St. George's respiratory questionnaire: MCID. COPD 2005;2:75-9.

29. French CT, Irwin RS, Fletcher KE, et al. Evaluation of a cough-specific quality-of-life questionnaire. Chest 2002;121:1123-31.

30. Majid A, Alape D, Kheir F, et al. Short-term use of uncovered self-expanding metallic airway stents for severe expiratory central airway collapse. Respiration 2016;92:389-96.

31. Tashkin DP, Celli B, Senn S, et al. A 4-year trial of tiotropium in chronic obstructive pulmonary disease. $\mathrm{N}$ Engl J Med 2008;359:1543-54.

32. Calverley PM, Anderson JA, Celli B, et al. Salmeterol and fluticasone propionate and survival in chronic obstructive pulmonary disease. N Engl J Med 2007;356:775-89. 